\title{
A NOTE ON JACOBSON RINGS AND POLYNOMIAL RINGS
}

\author{
MIGUEL FERRERO AND MICHAEL M. PARMENTER
}

(Communicated by Donald S. Passman)

\begin{abstract}
As is well known, if $R$ is a ring in which every prime ideal is an intersection of primitive ideals, the same is true of $R[X]$. The purpose of this paper is to give a general theorem which shows that the above result remains true when many other classes of prime ideals are considered in place of primitive ideals.
\end{abstract}

\section{INTRODUCTION}

Throughout this paper we assume that $R$ is a ring with identity element and $R[X]$ is the polynomial ring over $R$ in an indeterminate $X$. A ring $R$ is said to be a Jacobson ring if every prime ideal of $R$ is an intersection of primitive (either left or right) ideals. In [7], Watters proved that if $R$ is a Jacobson ring, the polynomial ring $R[X]$ is also a Jacobson ring. A similar result also holds for Brown-McCoy rings [8], i.e., rings in which every prime ideal is an intersection of maximal ideals.

In this note, $\mathscr{A}$ will always denote a class of prime rings. We say that an ideal $P$ of $R$ is an $\mathscr{A}$-ideal if $R / P \in \mathscr{A}$. When every prime ideal of $R$ is an intersection of $\mathscr{A}$-ideals, the ring $R$ is said to be an $\mathscr{A}$-Jacobson ring. For example, if $\mathscr{A}$ is the class of primitive (simple) rings, then an $\mathscr{A}$-Jacobson ring is a Jacobson (Brown-McCoy) ring.

The main purpose of this paper is to prove the following

Theorem 5. Assume that $\mathscr{A}$ is a class of prime rings satisfying condition (A). If $R$ is an $\mathscr{A}$-Jacobson ring, then so is $R[X]$.

Condition $(\mathrm{A})$ is defined near the beginning of $\S 2$. Since primitive (simple) rings satisfy this condition, the above theorem includes as particular cases the results in [7 and 8]. However, we show that many other classes of prime rings satisfy condition (A) as well. Some examples include prime Noetherian rings,

Received by the editors December 10, 1987 and, in revised form, February 22, 1988.

1980 Mathematics Subject Classification (1985 Revision). Primary 16A12, 16A66; Secondary $16 \mathrm{~A} 10$.

The research of the first author was supported by Conselho Nacional de Desenvolvimento Cientifico e Tecnológico (CNPq), Brazil, and Memorial University of Newfoundland.

The research of the second author was supported by NSERC grant A-8775. 
strongly prime rings, prime nonsingular rings and prime Goldie rings. These cases, as well as others, are studied in the latter part of $\S 2$.

Independent of the above, we offer in $\S 1$ a short direct proof of the Jacobson ring result from [7]. Our purpose here is twofold. First, the argument differs from that of Watters and we feel it should be noted somewhere. Secondly, some of the ideas in $\S 1$ are also required in $\S 2$.

Let us set some notation and terminology. If $I$ is an ideal (right ideal) of $R[X]$, then by $\tau(I)$ we denote the ideal (right ideal) of $R$ consisting of 0 and all the leading coefficients of all the polynomials of minimal degree in $I$. For $f \in R[X], \partial f$ denotes the degree of $f$ and $\operatorname{lc}(f)$ the leading coefficient of $f$. We set

$$
\operatorname{Min}(I)=\operatorname{Min}\{\partial f: 0 \neq f \in I\} .
$$

Finally, an ideal $P$ of $R[X]$ will be called $R$-disjoint if $P \neq 0$ and $P \cap R=0$.

\section{JACOBSON RINGS}

In this section we will give a brief argument showing that if $R$ is a Jacobson ring, then $R[X]$ is also a Jacobson ring. Crucial to our proof is the following lemma, which will be required also in $\S 2$. We are indebted to the referee for pointing out that this lemma is due to Bergman (unpublished) and that published proofs have appeared in several places (e.g. [6, Corollary 2.13]).

Lemma 1. Let $R$ be a prime ring and $P$ an $R$-disjoint ideal of $R[X]$. The following are equivalent:

(i) $P$ is a prime ideal of $R[X]$.

(ii) $P$ is maximal in the set of $R$-disjoint ideals of $R[X]$.

We now prove the result.

Proposition 2 (cf. [7]). If $R$ is a Jacobson ring, then $R[X]$ is a Jacobson ring. Proof. Let $P$ be a prime ideal of $R[X]$. By factoring out $P \cap R$ we may assume that $P \cap R=0$. We wish to prove that $J(R[X] / P)=0$.

If $P=0$, then $J(R[X] / P)=J(R[X]) \subseteq J(R)[X]=0[1]$, since $J(R)=0$ by the assumption.

Assume that $P \neq 0$ and $J(R[X] / P)=I / P \neq 0$. By Lemma 1 , we must have $I \cap R \neq 0$. We claim that $(I \cap R) \tau(P) \subseteq J(R)$. Once this is established, the proof is complete by contradiction, since $J(R)=0, \tau(P) \neq 0$ and $R$ is prime. From this point on, our argument differs from that of Watters.

Let $a \in(I \cap R) \tau(P)$. Since $a \in I$, we must have $a+g+a g \in P$ for some $g \in R[X]$. This means that $g=a(-g-1)+p$ for some $p \in P$, so $a+h+a h \in P$ where $h=a(-g-1) \in \tau(P)[X]$ and $\partial h \leq \partial g$. If $\partial h<\operatorname{Min}(P)$ then we must have $a+c+a c=0$ where $c$ is the constant term in $h$.

If $\partial h=m \geq \operatorname{Min}(P)=n$, then let $f$ be a polynomial of minimal degree in $P$ which has the same leading coefficient as $h$. Consider $k=h-X^{m-n} f$ and note that $\partial k<\partial h$ and $a+k+a k \equiv a+h+a h(\bmod P)$. Continuing this reduction we obtain a right quasi-inverse for a modulo $P$, which is of degree 
less than $n$. So as above we have that $a$ is right quasi-invertible in $R$. The proof is complete.

\section{MAIN RESUltS}

We begin this section with the following key lemma.

Lemma 3. Assume that $P$ is an $R$-disjoint prime ideal of $R[X]$ and $Q$ is a nonzero prime ideal or a maximal right ideal of $R$. If $\tau(P) \nsubseteq Q$, then $(P+Q[X]) \cap R=Q$.

Proof. Assume to the contrary that there exists $r \in R-Q$ such that $r=h_{1}+h_{2}$, for some $h_{1} \in P$ and $h_{2} \in Q[X]$. It follows that there exists $g=a_{m} X^{m}+$ $\cdots+a_{0} \in P$ with $a_{i} \in Q, i \geq 1$ and $a_{0} \notin Q$. Take such a $g$ of minimal degree with respect to these conditions and suppose that $f=b_{n} X^{n}+\cdots+$ $b_{0}$ is a polynomial of minimal degree in $P$. If $b_{n} \notin Q$, under either of the assumptions there exists $c \in R$ such that $a_{0} c b_{n} \notin Q$. Hence $g c b_{n}-$ $X^{m-n} a_{m} c f \in P$, which contradicts the minimality of $\partial g$.

A class $\mathscr{A}$ of prime rings is said to satisfy condition (A) if the following holds:

(A) If $R \in \mathscr{A}$, then $R[X] / P \in \mathscr{A}$ for every $R$-disjoint prime ideal $P$ of $R[X]$.

It is clear using Lemma 1 that the class of simple rings satisfies (A), and we shall show in Proposition 7 that the class of primitive rings satisfies (A) as well. Hence Theorem 5 is indeed a generalization of results in [7 and 8]. However, there are many other classes in which (A) holds. For some of these, the proof that $(A)$ is satisfied is very easy, for example prime Noetherian rings, simple Artinian rings, fields, finite fields, prime rings which are nil (locally nilpotent) semisimple and $G$-rings. For other classes, the verification of condition (A) is less obvious, and will be given later on.

We require one more lemma. Denote by $\mathscr{A}(R)$ the intersection of all prime ideals $P$ of $R$ such that $R / P \in \mathscr{A}$.

Lemma 4. Assume that $\mathscr{A}$ satisfies condition (A) and $R \in \mathscr{A}$. Then $\mathscr{A}(R[X])$ $=0$.

Proof. Let $Q$ be the Martindale ring of quotients of $R$ and $C$ the centre of $Q$. Then $C$ is a field because $R$ is prime. Take a monic irreducible polynomial $f \in C[X]$ and put $P_{f}^{*}=f Q[X]$. If $M$ is a maximal $Q$-disjoint ideal of $Q[X]$ with $M \supseteq P_{f}^{*}$ and $h=a_{n} X^{n}+\cdots+a_{0} \in M$ is a polynomial of minimal degree in $M$, we have $a_{n} q h-h q a_{n}=0$ for every $q \in Q$. Hence $a_{n} q a_{i}-a_{i} q a_{n}=0$ for all $q \in Q$ and $i=0, \ldots, n-1$. By [5, Lemma 1.3.2] there exists $c_{i} \in C$ such that $a_{i}=c_{i} a_{n}, i=0, \ldots, n-1$. Then $h=h_{0} a_{n}$ with $h_{0} \in C[X]$. Since $h_{0} Q[X] a_{n} \subseteq M$ and $M$ is prime by Lemma 1 , we have $h_{0} \in M$. If $g \in M$, then $g=p h_{0}+r$, where $p, r$ are in $Q[X]$ and $\partial r<n$. So $r=g-p h_{0} \in M$ and it follows that $r=0$. We get $M=h_{0} Q[X] \supseteq P_{f}^{*}$ and thus $f \in h_{0} Q[X]$. 
Since $f$ is irreducible, $f=h_{0}$ and so $P_{f}^{*}=M$ is a prime ideal. Hence $P_{f}=P_{f}^{*} \cap R[X]$ is also a prime ideal [3, Theorem 1.4] and $R$-disjoint. By the assumption, $R[X] / P_{f}$ is in $\mathscr{A}$ and since $\bigcap_{f} P_{f}=0$, the result follows.

Now we can prove our main result.

Proof of Theorem 5. Suppose that $P$ is a prime ideal of $R[X]$. By factoring out $P \cap R$, we may assume that $P \cap R=0$. Then $R$ is prime and by the assumption $0=\bigcap_{i \in L}\left\{Q_{i} \triangleleft R: R / Q_{i} \in \mathscr{A}\right\}$. By Lemma $4, Q_{i}[X]$ is an intersection of $\mathscr{A}$ ideals of $R[X]$, and since $\bigcap_{i \in L} Q_{i}[X]=0$, it follows that $\mathscr{A}(R[X])=0$. This takes care of the case $P=0$, so Lemma 1 tells us that we may assume that $P$ is an ideal maximal with respect to $P \cap R=0$.

Now, put $I=\left\{i \in L:\left(P+Q_{i}[X]\right) \cap R=Q_{i}\right\}$ and $J=L-I$. We have $0=\left(\bigcap_{i \in I} Q_{i}\right) \cap\left(\bigcap_{i \in J} Q_{i}\right)$ and so the primality of $R$ allows us to conclude that $\bigcap_{i \in I} Q_{i}=0$, since by Lemma $3, \bigcap_{i \in J} Q_{i} \supseteq \tau(P) \neq 0$. For every $i \in I$, choose an ideal $P_{i}$ of $R[X]$ maximal with respect to $P_{i} \supseteq P+Q_{i}[X]$ and $P_{i} \cap R=Q_{i}$. Condition (A) tells us that $R[X] / P_{i} \in \mathscr{A}$ and since $\left(\bigcap_{i \in I} P_{i}\right) \cap R=\bigcap_{i \in I} Q_{i}=0$ and $P \subseteq \bigcap_{i \in I} P_{i}$, we conclude that $P=\bigcap_{i \in I} P_{i}$ as required.

Remark 1. Condition (A) could be weakened by requiring that $\mathscr{A}(R[X] / P)=0$ instead of $R[X] / P \in \mathscr{A}$ for every $R$-disjoint prime ideal $P$ of $R[X]$. With this apparently weaker condition, the above proof still holds, but we do not know any natural example of a class which satisfies this condition but not condition (A).

Remark 2. The essential difference between our proof of Theorem 5 and the original argument of Watters in [7, Lemma 4] is that Lemma 3 allows us to apply the going up argument to $Q_{i}$, not only when $Q_{i}$ is a maximal right ideal but also when $Q_{i}$ is a prime ideal.

Earlier in this section, we noted a number of classes of prime rings for which condition (A) trivially holds. Now we will verify (A) for less obvious cases.

Proposition 6. The following classes of prime rings satisfy condition (A):

(i) (right) prime nonsingular rings,

(ii) (right) strongly prime rings.

Proof. These cases are verified in [3, (Lemmas 3.5 and 3.2)]. However, for the sake of completeness, we will present here a short argument, different from that in [3], for the case (i). We note that case (ii) could be handled similarly.

Assume that $R$ is a prime nonsingular ring and let $P$ be an $R$-disjoint prime ideal of $R[X]$. By way of contradiction we assume that the (right) singular ideal $Z(R[X] / P)=I / P \neq 0$. By Lemma $1, I \cap R \neq 0$ and we may choose $0 \neq a \in I \cap R$. It is enough to see that $a \in Z(R)$.

Let $J$ be a nonzero right ideal of $R$. Then $J[X]$ is a right ideal of $R[X]$ and so there exists $g \in J[X]$ such that $g \notin P$ and $a g \in P$. Take such a $g$ of minimal degree $m$. If $m<\operatorname{Min}(P), a g=0$ and $a g_{i}=0$ for any nonzero coefficient of $g$. Since $g_{i} \in J$ we are done if we show that $m \geq \operatorname{Min}(P)$ is not possible. In fact, in this case choose a nonzero polynomial of minimal degree 
$n$ in $P$ and let $\operatorname{lc}(g)=g_{m}$ and $\operatorname{lc}(f)=f_{n}$. Since $g \notin P$, there exists $r \in R$ with $g r f_{n} \notin P$. This means that $g^{\prime}=g r f_{n}-g_{m} r f X^{m-n} \notin P$. But $\partial g^{\prime}<\partial g$ and $a g^{\prime} \in P$, which is a contradiction.

Proposition 7. The class of (right) primitive rings satisfies condition (A).

Proof. Let $P$ be an $R$-disjoint prime ideal of $R[X]$ and let $Q$ be a maximal right ideal of $R$ with $(Q: R)=\{r \in R: R r \subseteq Q\}=0$. It follows that $\tau(P) \nsubseteq Q$ and $(P+Q[X]) \cap R=Q$ by Lemma 3. Take a right ideal $M$ of $R[X]$ maximal with respect to $M \supseteq P+Q[X]$ and $M \cap R=Q$. Then $M$ is a maximal right ideal of $R[X]$ such that $(M: R[X]) \cap R=(Q: R)=0$. Since $(M: R[X]) \supseteq P$ it follows that $(M: R[X])=P$ and $P$ is primitive.

Finally, we show that the class of prime rings with finite (right) Goldie dimension also satisfies (A). First we need the following

Lemma 8. Suppose that $P$ is an $R$-disjoint prime ideal of $R[X]$ and $I \supseteq P$ is a right ideal such that $\operatorname{Min}(I)=\operatorname{Min}(P)$. Then $I=P$.

Proof. Let $f \in P$ be a polynomial of minimal degree $n \geq 1$ with $\operatorname{lc}(f)=a$. If $g=b_{n} X^{n}+\cdots+b_{0}$ is of minimal degree in $I$, then $g r a-b_{n} r f \in I$ for every $r \in R$. Minimality tells us that $g r a-b_{n} r f=0$, so $g R[X] a \subseteq P$ and hence $g \in P$. Now, if $h \in I$, induction on $\partial h$ gives us that $h \in P$.

Proposition 9. The class of prime rings with finite (right) Goldie dimension satisfies (A).

Proof. Let $P$ be an $R$-disjoint prime ideal of $R[X]$ and suppose that $\left(I_{i}\right)_{i \in I}$ is an infinite family of right ideals of $R[X]$ with $I_{i} \supset P$ for all $i$ and $\sum_{i \in I}\left(I_{i} / P\right)$ $=\sum_{i \in I} \bigoplus\left(I_{i} / P\right)$. We will reach a contradiction.

We have $\operatorname{Min}\left(I_{i}\right)<\operatorname{Min}(P)$ for each $i \in I$, by Lemma 8. So there exists an infinite subfamily for which the values of $\operatorname{Min}\left(I_{i}\right)$ are all equal. We relabel this subfamily as $I_{1}, I_{2}, \ldots$ Hence $\operatorname{Min}\left(I_{1}\right)=\operatorname{Min}\left(I_{2}\right)=\cdots<\operatorname{Min}(P)$. Put $s=\operatorname{Min}\left(I_{i}\right), i=1,2, \ldots$

Case I. Assume that there exists an integer $m$ such that $\operatorname{Min}\left(I_{m}\right)=$ $\operatorname{Min}\left(I_{m}+I_{m+1}\right)=\cdots=\operatorname{Min}\left(I_{m}+\cdots+I_{m+n}\right)$, for all $n$. In this case consider the right ideals $\tau\left(I_{m}\right), \tau\left(I_{m+1}\right), \ldots$ of $R$. By again relabelling, we may assume there exists $0 \neq a_{1} \in \tau\left(I_{1}\right) \cap \sum_{i=2}^{t} \tau\left(I_{i}\right)$ since $R$ is of finite Goldie dimension. Hence $a_{1}=a_{2}+\cdots+a_{t}$ where $a_{j} \in \tau\left(I_{j}\right)$ for $1 \leq j \leq t$. Since $\operatorname{Min}\left(I_{i}\right)=s$ for all $i$, there must exist polynomials $f_{i}=a_{i} X^{s}+h_{i} \in I_{i}$, where $\partial h_{i}<s$. Since $g=f_{1}-\left(f_{2}+\cdots+f_{t}\right) \in I_{1}+\cdots+I_{t}$ and $\partial g<s$, we have $g=0$. Hence $f_{1}=f_{2}+\cdots+f_{t} \in I_{1} \cap \sum_{j=2}^{t} I_{j}=P$ and hence $f_{1}=0$. This contradicts $a_{1} \neq 0$, so the result is established in this case.

Case II. We suppose that for every $m \geq 1$ there exists some $n>m$ such that $\operatorname{Min}\left(I_{m}+\cdots+I^{m+n}\right)<\operatorname{Min}\left(I_{m}\right)$. So there exists $j \geq 2$ such that $\operatorname{Min}\left(I_{1}+\cdots+I_{j}\right)<s$. Put $H_{1}=I_{1}+\cdots+I_{j}$ and consider $I_{k}^{\prime}=I_{j+k}$ for $k \geq 1$. Hence there exists $t \geq 1$ such that $\operatorname{Min}\left(I_{1}^{\prime}+\cdots+I_{t}^{\prime}\right)<\operatorname{Min}\left(I_{1}^{\prime}\right)=s$ and 
write $H_{2}=I_{1_{\infty}}^{\prime}+\cdots+I_{t_{\infty}}^{\prime}$. By induction we form an infinite sequence $\left\{H_{i}\right\}$ such that $\sum_{i=1}^{\infty^{\infty}} H_{i} / P=\sum_{i=1}^{\infty} \bigoplus\left(H_{i} / P\right)$ and such that $\operatorname{Min}\left(H_{i}\right)<s$.

Now, we repeat the entire procedure with the $\left\{H_{i}\right\}$, either reaching a contradiction by Case I or reducing the degree again. But if we have reduced the degree to $\operatorname{Min}\left(H_{i}\right)=0$, then we are done because $R$ has finite Goldie dimension and $\sum_{i=1}^{\infty}\left(H_{i} \cap R\right)=\sum_{i=1}^{\infty} \bigoplus\left(H_{i} \cap R\right)$ in this case.

The following case is already contained in the literature [2, Lemma 2.3]. However, it is also a direct consequence of Proposition 6(i) and Proposition 9.

Corollary 10. The class of prime right Goldie rings satisfies condition (A).

Proof. It is enough to recall that a prime ring is right Goldie if and only if it is nonsingular and has finite Goldie dimension [4, Corollary 3.32].

Remark 1 . There are dual proofs, of course, for left conditions, i.e., left strongly prime rings, etc.

Remark 2. We would like to thank the referee for pointing out that the class of completely prime rings and the class of division rings both fail to satisfy condition (A). This can be seen by taking $R$ to be the division ring of real quaternions and $P$ the prime ideal $\left(X^{2}+1\right)$, since $R[X] / P \cong M_{2}(\mathbf{C})$.

Remark 3. We were unable to decide whether the following classes of prime rings satisfy condition (A).

(i) prime (von Neumann) regular rings,

(ii) prime rings with no nonzero (von Neumann) regular ideals,

(iii) prime rings which satisfy ACC on right annihilators.

Added in Proof. The results of [3] will appear in another paper by the author elsewhere.

\section{REFERENCES}

1. S. A. Amitsur, Radicals of polynomial rings, Canad. J. Math. 8 (1956), 355-361.

2. A. D. Bell, When are all prime ideals in an Ore extension Goldie?, Comm. Algebra 13 (1985), 1743-62.

3. M. Ferrero, Prime ideals in polynomial rings, preprint.

4. K. R. Goodearl, Ring theory, nonsingular rings and modules, Marcel Dekker, New York, 1976.

5. I. N. Herstein, Rings with involutions, Univ. of Chicago Press, Chicago and London, 1976.

6. K. R. Pearson, W. Stephenson and J. F. Watters, Skew polynomials and Jacobson rings, Proc. London Math. Soc. 42 (1981), 559-576.

7. J. F. Watters, Polynomial extensions of Jacobson rings, J. Algebra 36 (1975), 302-308.

8. __ The Brown-McCoy radical and Jacobson rings, Bull. Acad. Polon. Sci. Ser. Sci. Math. Astronom. Phys. 24 (1976), 91-99.

Instituto de Matematicas, Universidade Federal do Rio Grande de Sul, 90049-Porto ALEGRE, RS, BRASIL

Department of Mathematics and Statistics, Memorial University of Newfoundland, St. John's, Newfoundland, A1C 5S7, Canada 